\title{
Neuroimaging in idiopathic adult-onset focal dystonia
}

\author{
Giovanni Fabbrini ${ }^{1,2}$ (D) Antonella Conte ${ }^{1,2} \cdot$ Gina Ferrazzano $^{1} \cdot$ Marcello Esposito $^{3} \cdot$ Alberto Albanese $^{4}$. \\ Roberta Pellicciari ${ }^{5}$. Francesca Di Biasio ${ }^{6}$. Francesco Bono ${ }^{7}$. Roberto Eleopra ${ }^{8}$. Tommaso Ercoli ${ }^{9}$. \\ Maria Concetta Altavista ${ }^{10} \cdot$ Alfredo Berardelli $^{1,2} \cdot$ Giovanni Defazio $^{9} \cdot$ The Italian Dystonia Registry participants $^{2}$
}

Received: 7 May 2020 / Accepted: 23 December 2020

(C) Fondazione Società Italiana di Neurologia 2021

\begin{abstract}
We aimed to study the attitude of Italian neurologists in the use of conventional MRI in patients with idiopathic adult-onset focal dystonia. Patients were included in the Italian Dystonia Registry by experts working in different Italian centers. MRI was available for 1045 of the $1471(71 \%)$ patients included in the analysis. Using logistic regression analysis, we found that MRI was more likely to be performed in patients with cervical dystonia, spasmodic dysphonia, or non-task-specific upper limb dystonia, whereas it was less likely to be performed in patients with blepharospasm or task-specific upper limb dystonia. We did not find differences in the number of MRIs performed between neurological centers in Northern, Central, and Southern Italy. We conclude that although the diagnosis of idiopathic adult-onset dystonia is mainly based on clinical grounds, many movement disorder experts rely on MRI to confirm a diagnosis of idiopathic dystonia. We suggest that neuroimaging should be used in patients with adult-onset focal dystonia to rule out secondary forms.
\end{abstract}

Keywords Blepharospasm $\cdot$ Cervical dystonia $\cdot$ Laryngeal dystonia $\cdot$ Arm dystonia $\cdot$ Magnetic resonance imaging

\section{Introduction}

Idiopathic focal dystonias are clinical entities characterized by the presence of involuntary movements and postures in focal segments of the body [1]. Blepharospasm (BSP), cervical dystonia (CD), spasmodic dysphonia (SD), and upper limb dystonia (ULD) are the most frequent idiopathic adult-onset focal dystonias (AOFDs) [2]. Diagnosis of AOFD forms is usually based on history and clinical examination. In AOFD, conventional neuroimaging studies are usually normal [3]. Although some experts maintain that MRI studies are not necessary in most AOFDs [4], other experts suggest that a normal MRI or $\mathrm{CT}$ is useful to rule out symptomatic dystonia forms [5-7].

Giovanni Fabbrini

Giovanni.fabbrini@uniroma1.it

1 Department of Human Neurosciences, Sapienza University of Rome, Viale dell'Università 30, 00185 Rome, Italy

2 IRCCS Neuromed, Pozzilli, Italy

3 Clinical Neurophysiology Unit "Esposito Marcello", Cardarelli Hospital, Naples, Italy

4 Department of Neurology, IRCCS, Humanitas Research Hospital, Rozzano, Milan, Italy

The Italian Dystonia Registry (IDR) is a multicenter data storage system that collects the diagnostic and clinical data of patients with different types of dystonia from several specialized neurological centers throughout Italy [8-11]. The IDR questionnaire also includes a question on whether patients had undergone an MRI examination, and, if so, whether it identified lesions in the basal ganglia, thalamus, or other structures possibly involved in symptomatic dystonia [12-14]. In order to see whether Italian movement disorder specialists consider neuroimaging necessary in idiopathic AOFD patients, we analyzed whether these patients underwent MRI examination. We also evaluated whether the use of MRI in diagnosing AOFD differed between centers located in Moro University of Bari, Bari, Italy

6 San Martino Polyclinic Hospital IRCCS, Genoa, Italy

7 Botulinum Toxin Center, Neurology Unit A.O.U. Mater domini, Catanzaro, Italy

8 Parkinson and Movement Disorders Unit, IRCCS Foundation, Neurology Institute "Carlo Besta”, Milan, Italy

9 Department of Medical Science and Public Health, Institute of Neurology, University of Cagliari, Cagliari, Italy

10 Neurology Unit, San Filippo Neri Hospital ASL Roma 1, Rome, Italy 
Northern, Central, and Southern Italy. This information may be useful to understand the approach Italian neurologists take when diagnosing AOFD.

\section{Methods}

The Italian Dystonia Registry was started on January 2016, and methods of data collection for the IDR are described elsewhere [8]. In the analysis, we included patients with a diagnosis of idiopathic AOFD, namely BSP, CD, ULD, and SD. Patients with a diagnosis of acquired dystonia, dystonia due to known structural causes, or with a confirmed genetic mutation were excluded from the study. We included all patients present in the IDR on November 30, 2019. We analyzed MRI examinations performed in the overall IDR population and in the different dystonia patient subgroups. Using a logistic regression model, we evaluated the clinical and demographic variables significantly associated with MRI for the patients enrolled in the IDR (dependent variable). Independent variables included type of dystonia, duration of symptoms, age, gender, and location of the center (Northern, Central, or Southern Italy). The study was approved by the ethics committees throughout Italy [8].

\section{Results}

The IDR included data from 1471 patients with idiopathic AOFD (508 males and 1008 females; mean age: 63.6 14.0 years; mean age of onset: $55.8 \pm 10.4$ years; mean disease duration: $8.6 \pm 9.2$ years). Of the 1471 dystonia patients enrolled, 572 came from Northern Italy, 538 from Central Italy, and 360 from Southern Italy. Clinical and demographic data of AOFD patients classified according to dystonia phenotype at onset are shown in Table 1.

MRI had been performed in 1045 of the 1471 patients included in the analysis (71\%), and on MRI, none of these patients showed lesions in brain areas possibly causing dystonia. The percentage of MRI performed in the various forms of dystonias is reported in Fig. 1. By stratifying dystonia patients according to the three geographical areas, we found that MRI had been performed in 422 patients (73.7\%) in Northern Italy, 396 patients $(73.6 \%)$ in Central Italy, and 227 patients $(63 \%)$ in Southern Italy.

Logistic regression analysis disclosed that MRI was more likely to be performed in CD, SD, and non-task-specific ULD patients than in BSP or task-specific ULD patients. The analysis also disclosed that the location of the centers was not associated with the number of MRI procedures (Table 2).

\section{Discussion}

IDR data showed that conventional MRI studies were performed in approximately $70 \%$ of Italian patients who had a diagnosis of idiopathic AOFD. CD, SD, and non-task-specific ULD patients were more likely to undergo MRI than patients with BSP or task-specific ULD. No significant differences were present in the number of patients who underwent MRI in the different geographical areas of Italy.

It is not clearly established whether MRI should always be performed in AOFD patients. Since dystonia due to structural lesions in the brain may be clinically indistinguishable from idiopathic dystonia [12-15], Mounier et al. [6] and European Federation of Neurological Societies (EFNS) guidelines [7] suggest that a normal MRI study is usually a prerequisite in order to determine whether dystonia is idiopathic. Conversely, Fung et al. [4] indicate that MRI is not necessary in most AOFDs and should probably be restricted to patients with early-onset dystonia or dystonia combined with other movement disorders. In our study, we found that about two-thirds of AOFD patients included in the IDR had undergone an MRI study. Our results suggest that although AOFD diagnosis is usually based on patient history and a complete clinical examination, Italian movement disorder experts perform MRI
Table 1 Clinical and demographic data of idiopathic adult-onset dystonia patients included in the Italian Dystonia Registry classified according to the site of dystonia onset

\begin{tabular}{llllll}
\hline $\begin{array}{l}\text { Dystonia } \\
\text { phenotype }\end{array}$ & $\begin{array}{l}\text { No. of } \\
\text { patients }\end{array}$ & $\begin{array}{l}\text { Gender } \\
\text { M/F }\end{array}$ & Age (Y) & $\begin{array}{l}\text { Age of onset } \\
(\mathrm{Y})\end{array}$ & $\begin{array}{l}\text { Symptom duration } \\
(\mathrm{Y})\end{array}$ \\
\hline BSP & 514 & $159 / 355$ & $69.4 \pm 10.6$ & $60.8 \pm 8.1$ & $8.2 \pm 8.6$ \\
CD & 662 & $234 / 128$ & $60.7 \pm 14.2$ & $54.4 \pm 12.7$ & $5.7 \pm 13.1$ \\
SD & 86 & $19 / 87$ & $67.9 \pm 12.5$ & $52.9 \pm 18.1$ & $15.6 \pm 12.1$ \\
ULD & 147 & $66 / 81$ & $56.2 \pm 16.8$ & $49.8 \pm 16.3$ & $7.8 \pm 16.1$ \\
$\begin{array}{l}\text { Task-specific } \\
\quad \text { ULD }\end{array}$ & 29 & $9 / 20$ & $51.5 \pm 17.4$ & $35.4 \pm 18.3$ & $17.9 \pm 17.2$ \\
LLD & 33 & $16 / 17$ & $52.8 \pm 18.3$ & $33.0 \pm 24.3$ & $15.5 \pm 16.4$ \\
\hline
\end{tabular}

Data are expressed as mean \pm standard deviation

$Y$ years, $B S P$ blepharospasm, $C D$ cervical dystonia, $S D$ spasmodic dysphonia, $U L D$ upper limb dystonia, $L L D$ lower limb dystonia 


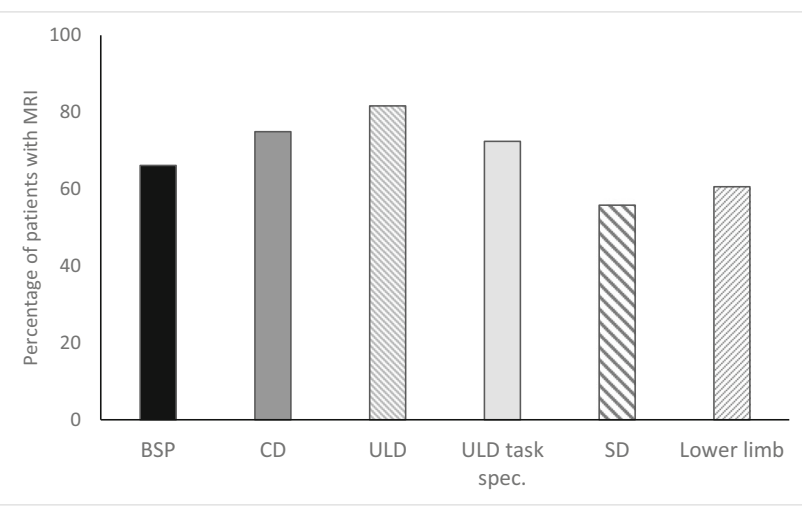

Fig. 1 Percentage of patients who had MRI according to the type of dystonia

studies in order to diagnose idiopathic dystonia and exclude symptomatic forms of dystonia associated with specific lesions in the brain.

Considering the various types of dystonia, logistic regression analysis disclosed that non-task-specific ULD, SD, and $\mathrm{CD}$ patients were more likely to undergo an MRI study than patients with BSP or task-specific ULD. The finding that patients with non-task-specific ULD were more likely to undergo MRI is consistent with the observation of Strader et al. [13] that the upper limbs were the most commonly affected body site in symptomatic dystonia due to brain lesions. The high rate of MRI in SD patients may be due to the observation that since SD is a rare condition, clinicians are often uncertain about the diagnosis [16]. MRI was also frequently performed in $\mathrm{CD}$ patients, possibly due to the desire to rule out brain or cervical spine lesions [12].

Conversely, MRI was performed less frequently in BSP and task-specific ULD patients. Possible reasons MRI was performed less frequently in these two forms of dystonia may be due to the fact that physicians consider it unlikely that structural lesions cause simultaneous involvement of bilateral

Table 2 Logistic regression analysis model showing demographic and clinical features associated with the dependent variable "MRI availability" (Yes $=1$, No $=0)$

\begin{tabular}{llll}
\hline Variable & Odds ratio & $95 \%$ CI & $p$ value \\
\hline Gender (female) & 1.02 & $0.86-1.22$ & 0.76 \\
Age & 0.99 & $0.98-1.00$ & 0.21 \\
Disease duration & 0.99 & $0.99-1.00$ & 0.38 \\
BSP & 1.04 & $0.75-1.43$ & 0.81 \\
CD & 1.44 & $1.02-2.04$ & 0.03 \\
SD & 0.52 & $0.32-0.85$ & 0.01 \\
Task-specific dystonia & 1.60 & $0.89-2.89$ & 0.11 \\
Non-task-specific ULD & 1.95 & $1.17-3.25$ & 0.009 \\
Tremor & 0.84 & $0.53-1.32$ & 0.45 \\
Geographical region & 1.00 & $0.88-1.15$ & 0.90 \\
\hline
\end{tabular}

structures, as in BSP, or that structural lesions, only rarely described in the brainstem, cause BSP [15]. In addition, structural lesions very rarely cause task-specific dystonia [3, 5]. The observation that MRI studies were performed with a similar frequency across the various geographical areas of Italy suggests that the number of MRIs performed is not conditioned by differences in regional healthcare policy, but more likely reflects the desire of Italian movement disorder experts to use conventional MRI to support a diagnosis made only on clinical grounds.

The strength of this study is that the analysis of MRIs performed included a large group of AOFD patients. A limitation is that patients enrolled in the IDR were followed in specialized centers, and therefore, the findings of this study cannot be generalized to the entire population.

In conclusion, a large percentage of movement disorder experts in Italy rely not only on clinical grounds but also on conventional MRI, to diagnose AOFD.

Acknowledgments The Italian Dystonia Registry participants are Stefania Lalli: Department of Neurology, IRCCS, Humanitas Research Hospital, Rozzano, Milan, Italy; Roberto Erro, Paolo Barone, and Sara Scannapieco: Department of Medicine, Surgery, and Dentistry "Scuola Medica Salernitana", Neuroscience Section, University of Salerno, Baronissi, Italy; Roberta Marchese: Department of Experimental Medicine, Section of Human Physiology, University of Genoa, Italy; Giulio Demonte and Domenico Santangelo: Botulinum Toxin Center, Neurology Unit A.O.U. Mater domini, Catanzaro, Italy; Laura Avanzino: Department of Experimental Medicine, Section of Human Physiology, University of Genoa, Italy; Grazia Devigili: Parkinson and Movement Disorders Unit, IRCCS Foundation, Neurology Institute "Carlo Besta", Milan, Italy; Valentina Durastanti: Neurology Unit, San Filippo Neri Hospital ASL Roma 1, Rome, Italy; Marinella Turla: Neurology Unit, ASST Valcamonica, Esine, Italy; Sonia Mazzucchi: Department of Clinical and Experimental Medicine, University of Pisa, Italy; Martina Petracca and Anna Rita Bentivoglio: Foundation Polyclinic Hospital A. Gemelli - IRCCS, Rome, Italy; Institute of Neurology, Catholic University of the Sacred Heart, Rome, Italy; Maurizio Zibetti: Department of Neuroscience "Rita Levi Montalcini," University of Turin, Turin, Italy; Laura Bertolasi: Neurology Unit, University Hospital, Verona, Italy; Maria Sofia Cotelli: Neurology Unit, ASST Valcamonica, Esine, Italy; Roberto Ceravolo: Department of Clinical and Experimental Medicine, University of Pisa, Italy; Cesa Scaglione: IRCCS Institute of Neurological Sciences, Bologna, Italy; Giovanni Cossu and Valentina Oppo: Neurological Service and Stroke Unit, Department of Neuroscience, AO Brotzu, Cagliari, Italy; Pierangelo Barbero: Neurology Unit, Umberto I Hospital, Turin, Italy; Paolo Girlanda and Francesca Morgante: Department of Clinical and Experimental Medicine, University of Messina, Italy; Mario Coletti Moja: Neurology Unit, Umberto I Hospital, Turin, Italy; Salvatore Misceo: Neurology Unit, San Paolo Hospital, Bari, Italy; Giulia Di Lazzaro and Antonio Pisani: Neurology Unit, Department of System Medicine, University of Rome Tor Vergata, Rome, Italy; Giovanna Squintani and Michele Tinazzi: Department of Neuroscience, Biomedicine, and Movement, University of Verona, Italy; Nicola Modugno: IRCCS Neuromed. Luca Maderna: Department of Neurology and Laboratory of Neuroscience, IRCCS, Italian Auxological Institute, Milan, Italy; Brigida Minafra: Parkinson's Disease and Movement Disorders Unit, IRCCS, Mondino Foundation, Pavia, Italy; Luca Magistrelli: Movement Disorders Center, Neurology Unit, Department of Translational Medicine, University of Eastern 
Piemonte, Novara, Italy; $\mathrm{PhD}$ Program in Clinical and Experimental Medicine and Medical Humanities, University of Insubria, Varese, Italy; Marcello Romano: Complex Operative Unit of Neurology, Azienda Ospedaliera Villa Sofia Cervello Palermo, Italy; Marco Aguggia: Neurology Unit, AOOR Villa Sofia Cervello, Palermo, Italy; Nicola Tambasco: Department of Neurology, Santa Maria della Misericordia Hospital, University of Perugia, Perugia, Italy; Anna Castagna: Recovery and Functional Re-education Operative Unit, IRCCS Santa Maria Nascente Foundation Don Gnocchi, Italy; and Daniela Cassano: Neurology Unit, Maria Vittoria Hospital, Turin, Italy.

\section{Compliance with ethical standards}

Conflict of interest The authors declare that they have no conflict of interest.

Ethical approval Ethical appproval has been obtained in all centers participating to the Italian Dystonia Registry.

Informed consent All particpating patients have given provided informed consent.

\section{References}

1. Jinnah HA, Berardelli A, Comella C (2013) The focal dystonias: current views and challenges for future research. Mov Disord 28: 926-943

2. Defazio G, Abbruzzese G, Livrea P, Berardelli A (2004) Epidemiology of primary dystonia. Lancet Neurol 3:673-678

3. Rutledge JN, Hilal DK, Silver AJ, Defendini R, Fahn S (1988) Magnetic resonance imaging of dystonic states. Adv Neurol 50: 265-275

4. Fung VSC, Jinnah HA, Bhatia K, Vidailhet M (2013) Assessment of patients with isolated or combined dystonia: an update on dystonia syndrome. Mov Disord 28:889-898

5. Albanese A, Bhatia K, Bressman SP et al (2013) Phenomenology and classification of dystonia: a consensus update. Mov Disord 28: 863-873

6. Meunier S, Leherici S, Garnero L, Vidailhet M (2003) Dystonia: lessons from brain mapping. Neuroscientist 9(1):76-81

7. Albanese A, Asmus F, Bhatia KP, Elia AE, Elibol B, Filippini G, Gasser T, Krauss JK, Nardocci N, Newton A, Valls-Solé J (2011) EFNS guidelines on diagnosis and treatment of dystonia. Eur $\mathrm{J}$ Neurol 18(1):5-18

8. Defazio G, Esposito M, Abbruzzese G, Scaglione CL, Fabbrini G, Ferrazzano G, Peluso S, Pellicciari R, Gigante AF, Cossu G, Arca R, Avanzino L, Bono F, Mazza MR, Bertolasi L, Bacchin R, Eleopra R, Lettieri C, Morgante F, Altavista MC, Polidori L, Liguori R, Misceo S, Squintani G, Tinazzi M, Ceravolo R, Unti
E, Magistrelli L, Coletti Moja M, Modugno N, Petracca M, Tambasco N, Cotelli MS, Aguggia M, Pisani A, Romano M, Zibetti M, Bentivoglio AR, Albanese A, Girlanda P, Berardelli A (2017) The Italian Dystonia Registry: rationale, design and preliminary findings. Neurol Sci 38:819-825

9. Defazio G, Albanese A, Pellicciari R, Scaglione CL, Esposito M, Morgante F, Abbruzzese G, Bentivoglio AR, Bono F, Coletti Moja M, Fabbrini G, Girlanda P, Lopiano L, Pacchetti C, Romano M, Fadda L, Berardelli A (2019) Expert recommendations for diagnosing cervical, oromandibular and limb dystonia. Neurol Sci 40(1): 89-95

10. Esposito M, Fabbrini G, Ferrazzano G, Berardelli A, Peluso S, Cesari U, Gigante AF, Bentivoglio AR, Petracca M, Erro R, Barone P, Schirinzi T, Eleopra R, Avanzino L, Romano M, Scaglione CL, Cossu G, Morgante F, Minafra B, Zibetti M, Coletti Moja M, Turla M, Fadda L, Defazio G (2018) Spread of dystonia in patients with idiopathic adult onset laringeal dystonia. Eur J Neurol 25(11):1341-1344

11. Defazio G, Fabbrini G, Erro R, Albanese A, Barone P, Zibetti M, Esposito M, Pellicciari R, Avanzino L, Bono F, Eleopra R, Bertolasi L, Altavista MC, Cotelli MS, Ceravolo R, Scaglione C, Bentivoglio AR, Cossu G, Coletti Moja M, Girlanda P, Misceo S, Pisani A, Mascia MM, Ercoli T, Tinazzi M, Maderna L, Minafra B, Magistrelli L, Romano M, Aguggia M, Tambasco N, Castagna A, Cassano D, Berardelli A, Ferrazzano G, Lalli S, Silvestre F, Manganelli F, di Biasio F, Marchese R, Demonte G, Santangelo D, Devigili G, Durastanti V, Turla M, Mazzucchi S, Petracca M, Oppo V, Barbero P, Morgante F, di Lazzaro G, Squintani G, Modugno N (2020) Does acute peripheral trauma contribute to idiopathic adult-onset dystonia? Parkinsonism Relat Disord 71: 40-43

12. LeDoux MS, Brady KA (2003) Secondary cervical dystonia associated with structural lesions of the central nervous system. Mov Disord 18:60-69

13. Strader S, Rodnitzky RL, Gonzalez-Alegre P (2011) Secondary dystonia in a Botulinum Toxin Clinic: clinical characteristics, neuroanatomical substrate and comparison with idiopathic dystonia. Parkinsonism Relat Disord 17(10):749-752

14. Marsden CD, Obeso JA, Zarranz JJ, Lang AE (1985) The anatomical basis of symptomatic hemidystonia. Brain 108:463-483

15. Khooshnoodi MA, Factor SA, Jinnah HA (2013) Secondary blepharospasm associated with structural lesion of the brain. J Neurol Sci 331(1-2):98-101

16. Ludlow CL, Domanque R, Sharma D et al (2018) Consensus-based attributes for identifying patients with spasmodic dysphonia and other voice disorders. JAMA Otolarynol Head Neck Surg 144(8): $657-665$

Publisher's note Springer Nature remains neutral with regard to jurisdictional claims in published maps and institutional affiliations. 